SV

\title{
APPROXIMATE ANALYTICAL SOLUTIONS FOR PRIMARY CHATTER IN THE NON-LINEAR METAL CUTTING MODEL
}

\author{
J. WARMIŃSKI AND G. LITAK \\ Department of Applied Mechanics, Technical University of Lublin, Nadbystrzycka 36, \\ Lublin 20-618, Poland \\ M. P. CARTMEll
}

Department of Mechanical Engineering, James Watt Building, University of Glasgow, Glasgow, G128QQ, Scotland, UK. E-mail: matthewc@mech.gla.ac.uk

\section{R. KHANIN}

Department of Applied Mathematics and Theoretical Physics, University of Cambridge, Silver St., Cambridge, CB3 9EW, England, UK

AND

\section{WIERCIGROCH}

Centre for Applied Dynamics Research, Department of Engineering, Fraser Noble Building, King's College, University of Aberdeen, Aberdeen, AB24 3UE, Scotland, UK

(Received 2 October 2001, and in final form 8 April 2002)

This paper considers an accepted model of the metal cutting process dynamics in the context of an approximate analysis of the resulting non-linear differential equations of motion. The process model is based upon the established mechanics of orthogonal cutting and results in a pair of non-linear ordinary differential equations which are then restated in a form suitable for approximate analytical solution. The chosen solution technique is the perturbation method of multiple time scales and approximate closed-form solutions are generated for the most important non-resonant case. Numerical data are then substituted into the analytical solutions and key results are obtained and presented. Some comparisons between the exact numerical calculations for the forces involved and their reduced and simplified analytical counterparts are given. It is shown that there is almost no discernible difference between the two thus confirming the validity of the excitation functions adopted in the analysis for the data sets used, these being chosen to represent a real orthogonal cutting process. In an attempt to provide guidance for the selection of technological parameters for the avoidance of primary chatter, this paper determines for the first time the stability regions in terms of the depth of cut and the cutting speed co-ordinates.

(C) 2002 Elsevier Science Ltd. All rights reserved.

\section{INTRODUCTION}

The mechanics of metal cutting has been of interest to a large number of researchers for some considerable time and early models for the intermittent cutting process were proposed by Tobias [1], Opitz and Bernardi [2], and Tlusty [3]. These models were useful in that they attempted to incorporate the necessary physical interactions between the 
mechanics of the machine tool and the actual cutting process itself. Grabec [4, 5] provided considerable insight into this interactive approach to the modelling of self-excited chatter vibrations by providing useful numerical solutions and interpretations of practical problem data. Marui et al. [6], Lin and Weng [7, 8], Gradisek et al. [9-11], Stepan and Kalmar-Nagy [12], Litak et al. [13], Przystupa and Litak [14], Johnson and Moon [15], Stepan [16], Wiercigroch and Budak [17], and Moon and Kalmar-Nagy [18] carried out further work on this general theme. Rabinowicz [19], Bell and Burdekin [20] and Wiercigroch [21, 22] considered an additional phenomenological issue, in the form of stick-slip effects observable in the machine tool slide-way.

Chaotic motions have been observed in the responses of chattering machine-tool systems by Grabec [4, 5] and Wiercigroch [22, 23], Wiercigroch and Cheng [24], Warmiński et al. [25], Kalmar-Nagy and Pratt [26], and Wiercigroch and Krivtsov [27]. These authors have examined the dynamics of several different variations on the basic tool-to-work-piece configuration. The general conclusion to be drawn from their work is that intermittency (i.e., chatter vibration) is consistently seen to be responsible for unstable and bifurcatory behaviour for certain sets of design data. In other words, it seems possible to find practical design data for a large variety of models and systems which will lead, ultimately, to instability, and to bifurcations leading to chaos. This in itself is an important finding because it indicates that the metal cutting process is potentially relatively difficult to control, and that stable cutting cannot necessarily be guaranteed for all system designs. It also implies the possibility of critical performance degradation in certain cases of metal cutting. Practically speaking, stick-slip and intermittency effects may arise because of deviations in the work-piece, the jamming up of chip formation, or dynamical delay effects due to chip thickness variation causing unevenness in the work-piece surface.

Approximate analytical solutions have been proposed for modelling different aspects of the non-linear dynamical behaviour of the cutting process in recent years. For example, a non-linear one-degree-of-freedom cutting system has been investigated by Nosyreva and Molinari [28] and analytical solutions were obtained by applying the multiple scales method. In this work the main influence was of the cutting velocity on the mean friction coefficient, thereby reflecting the mechanics of the Merchant model [29]. The problem was reduced to a consideration of motion in the direction perpendicular to the cutting velocity. In addition to this the influence of the ploughing force on the system dynamics was analyzed, showing that for some supercritical conditions the amplitude of vibration saturates due to non-linear effects. This has been confirmed in a slightly different model by Wiercigroch and Krivtsov [27], for the case where the discontinuity of the friction characteristics is responsible for bounding the oscillations. A two-dimensional dynamical investigation of orthogonal cutting was carried out by Hwang et al. [30], in which a simple model of chip formation and the regenerative cutting effects was analyzed using the multiple scales method. This approach considered the system under investigation to be weakly non-linear and therefore assessed the influence of strong non-linearities by direct numerical integration. This numerical study revealed that a different cutting thicknesses can result in qualitatively different behaviour of the system. A non-linear single-degree-of-freedom model originally proposed by Hanna and Tobias [31] has been discussed by Nayfeh et al. [32]. This model included linear structural damping, quadratic and cubic non-linear stiffness of the machine tool, and linear, quadratic and cubic regenerative terms, as required to define the regenerative cutting process in dynamical terms. The method of multiple scales and bifurcation analysis were both applied to the problem in order to predict the complex dynamics of the system. By using phase portraits, Poincaré sections, and power spectra, periodic, quasiperiodic, and chaotic attractors were constructed and the importance of including non-linear stiffness terms was also emphasized. 
As shown in the discussion above there is evidence of growing interest in the application of approximate analytical methods to support numerical analysis of the dynamic interactions occurring in cutting processes, and in particular, to provide guidance on how to select technological parameters in order avoid primary chatter vibrations. This paper looks, for the first time, at the stability regions in the context of the depth of cut and the cutting speed co-ordinates, and in order to achieve this the principal foundation of the work has been based on an investigation of the original dynamical model proposed by Wiercigroch [23], noting that this model has been derived from that of Grabec [4, 5]. In this paper, Wiercigroch's model has been developed to include the effects of Coulomb friction, but in the context of analytical rather than numerical solutions. Naturally, this pre-supposes the existence of periodic solution forms both for the general non-resonant condition as well as certain internal resonance conditions, but obviously will not deal with cases of non-periodic responses. The non-resonant condition is interesting because, in terms of the frequency domain, it lies between and around the various resonances that the multiple scales analysis also predicts. Therefore, a non-resonant response is a non-trivial vibration which is spread across the working frequency domain, but with the various resonances taking precedence at appropriate points in the domain. In this paper the nonresonant chatter vibration is analyzed to show conditions in which it can grow and vice versa. The main resonant cases are stated but not analyzed here. The perturbation technique of multiple scales is applied to the problem, after some manipulation of the basic system equations of motion. Bespoke computer algebra software is used to generate the perturbation equations (see references [33, 34]) and to provide the required analytical solutions to a pre-specified level of approximation accuracy. It is also shown that the analytically complicated equations of motion due to Wiercigroch [23] can be simplified somewhat without too much loss of generality or accuracy. This simplification applies specifically to the orthogonal cutting forces $f_{x}$ and $f_{y}$ and is discussed further within the following analysis.

\section{MODELLING OF SYSTEM DYNAMICS}

On the basis of the work of Juilian et al. [35], in which it was shown that there is inherent discontinuity in the metal cutting process, and the observation that there is feedback between the machine tool and the actual cutting process (in the form of a vector of cutting forces) Wiercigroch [23] proposed a revised version of the model originally suggested by Grabec [4, 5]. This revision to the original work of Grabec [4, 5] is discussed in detail in reference [23] in the form of additional frictional modelling. This is related to the work of Hastings et al. [36] in which it was shown that there is a physically based mathematical interdependence between the orthogonal cutting force terms, noting that these terms are made up of discontinuous frictional effects. The ensuing model is considered to be reasonably representative of the process for this reason and also because of its adoption of orthogonal machining whereby the cutting edge is parallel to the work-piece and normal to the cutting direction as shown in Figure 1.

The result of this process of model development is a two-degree-of-freedom representation in which Heaviside functions appear within the frictionally based force terms $f_{x}$ and $f_{y}$. These are necessary to represent the stick-slip discontinuity, as is the presence of the relative velocity $v_{r}$ between the tool and the work-piece in the $x$ direction (the direction of travel of the work-piece). The two degrees of freedom are represented by $x$ and $y$, which are non-dimensionalized generalized co-ordinates defining motion of the tool in the $x$ and the $y$ directions (the latter being axially along the tool). The tool is 


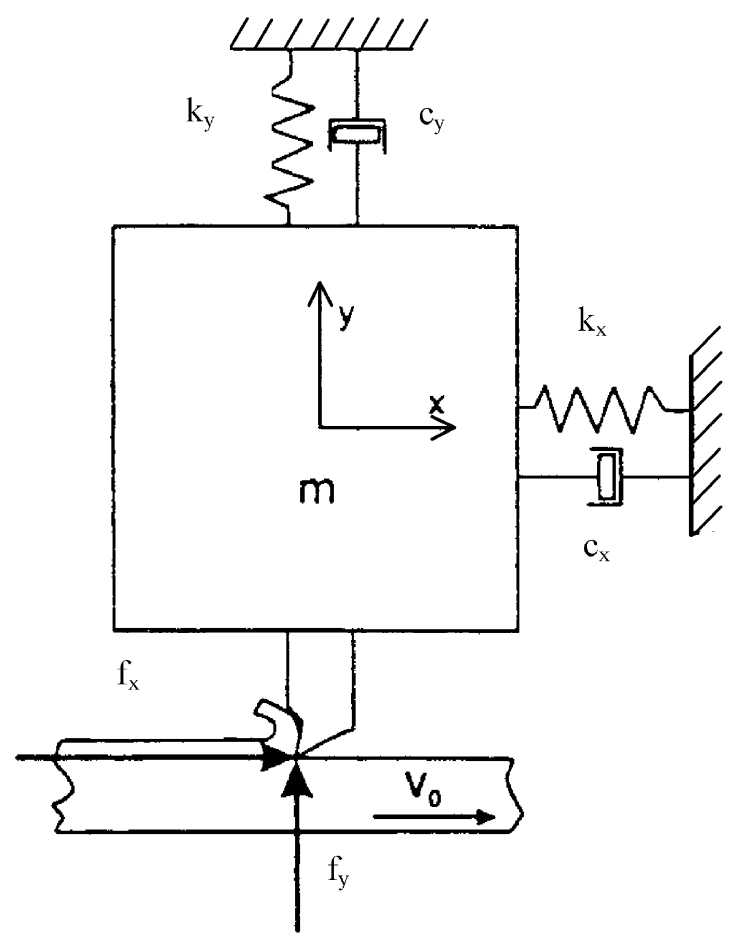

Figure 1. Schematic diagram of the orthogonal metal cutting process, according to Wiercigroch [23].

considered to be supported by means of foundation-mounted linear springs and dampers oriented in both directions, a relatively straightforward but not unrealistic assumption. In this way, the elastic and dissipative properties are defined for the tool and its mounting assembly. This is therefore a good definitive, and practically tractable, model for the process and in the form suggested by Wiercigroch [23] it can be seen that the equations of motion for the system are given as follows:

$$
\begin{aligned}
x^{\prime \prime}+2 \xi_{x} x^{\prime}+x= & q_{0} h\left[C_{1}\left(\left|v_{r}\right|-1\right)^{2}+1\right] \mathrm{H}(h)\left(\mathrm{H}\left(v_{r}\right) \frac{1}{1+\mu_{0}}+\operatorname{sgn}\left(v_{r}\right) \frac{\mu_{0}}{1+\mu_{0}}\right)=f_{x}, \quad(1) \\
y^{\prime \prime}+2 \xi_{y} \sqrt{\alpha} y^{\prime}+\alpha y= & {\left[C_{2}\left(\left|v_{f}\right|-1\right)^{2}+1\right]\left[C_{3}(h-1)^{2}+1\right] \mathrm{H}\left(f_{x}\right) \operatorname{sgn}\left(v_{f}\right) q_{0} h\left[C_{1}\left(\left|v_{r}\right|-1\right)^{2}+1\right] } \\
& \mathrm{H}(h)\left(\mathrm{H}\left(v_{r}\right) \frac{1}{1+\mu_{o}}+\operatorname{sgn}\left(v_{r}\right) \frac{\mu_{0}}{1+\mu_{0}}\right)=f_{y},
\end{aligned}
$$

The right-hand side terms in each equation collectively form the non-dimensionalized forces $f_{x}$ and $f_{y}$. It should also be noted that $q_{0}$ is the magnitude of the cutting force, $h_{0}$ is initial depth of cut, $\mu_{0}$ is the static friction coefficient, and that the relative velocity between the tool and the work-piece in the $x$ and $y$ directions, respectively, $v_{r}$ and $v_{f}$, and the actual depth of cut, $h$, are defined and related as follows, given that $v_{0}, R, R_{0}$ and $C_{1-4}$ are the absolute velocity of the work-piece, the coefficient of variable shear plastic deformation, the shear plastic deformation constant, and cutting process constants, thus, $v_{r}=v_{0}-x^{\prime}, \quad v_{f}=v_{0}-R y^{\prime}=v_{0}-R_{0}\left[C_{4}\left(v_{r}-1\right)^{2}+1\right] y^{\prime}=v_{0}-R_{0}\left[C_{4}\left(v_{0}-x^{\prime}-1\right)^{2}+1\right] y^{\prime}$, $h=h_{0}-y$. 
Equation (1) can be rewritten in simpler form by firstly substituting the forms given above for the relative velocities in $x$ and $y$ and the depth of cut $\left(v_{r}, v_{f}\right.$, and $\left.h\right)$ and then lumping together the terms that emerge from this so that the coefficients in the differential equation are simplified as far as possible, in the limit of small oscillations,

$$
x^{\prime \prime}+\left(2 \xi_{x}+\beta_{2}\right) x^{\prime}+x+\beta_{3} y-\beta_{1} x^{\prime 2}+\beta_{4} x^{\prime 2} y-\beta_{5} x^{\prime} y=Q_{2},
$$

where

$$
\begin{aligned}
& \beta_{1}=Q_{1} h_{0} C_{1}, \quad \beta_{2}=2 Q_{1} h_{o} C_{1}\left(v_{0}-1\right), \quad \beta_{3}=Q_{1} C_{1}\left[\left(v_{0}-1\right)^{2}+\frac{1}{C_{1}}\right], \\
& \beta_{4}=Q_{1} C_{1}, \quad \beta_{5}=2 Q_{1} C_{1}\left(v_{0}-1\right), \quad Q_{1}=q_{0}\left[\mathrm{H}\left(v_{r}\right) \frac{1}{1+\mu_{o}}+\operatorname{sgn}\left(v_{r}\right) \frac{\mu_{0}}{1+\mu_{0}}\right] \mathrm{H}(h), \\
& Q_{2}=Q_{1}\left[h_{0} C_{1} v_{0}^{2}-2 h_{0} C_{1} v_{0}+h_{0} C_{1}+h_{0}\right] .
\end{aligned}
$$

In the case of equation (2) certain groupings of constants can be replaced by simple substitutions to lead to a differential equation which is more explicit (note that this is also because of the use of the substitutions given above for $v_{r}, v_{f}$, and $h$ once again), therefore,

$$
\begin{aligned}
y^{\prime \prime}+2 \xi_{y} \sqrt{\alpha} y^{\prime}+\alpha y= & Q_{3}\left(h_{0}-y\right)\left[C_{1} \sigma_{2}+C_{1} x^{\prime 2}-2 C_{1} v_{0} x^{\prime}+2 C_{1} x^{\prime}+1\right]\left[C_{2} \delta_{1}^{2}-2 C_{2} \delta_{1} \delta_{2} x^{\prime 2} y^{\prime}\right. \\
& +2 C_{2} \delta_{1} \delta_{3} x^{\prime} y^{\prime}-2 C_{2} \delta_{1} \delta_{4} y^{\prime}+C_{2} \delta_{2}^{2} x^{\prime 4} y^{\prime 2}-2 C_{2} \delta_{2} \delta_{3} x^{\prime 3} y^{\prime 2} \\
& \left.+2 C_{2} \delta_{2} \delta_{4} x^{\prime 2} y^{\prime 2}+C_{2} \delta_{3}^{2} x^{\prime 2} y^{\prime 2}-2 C_{2} \delta_{3} \delta_{4} x^{\prime} y^{\prime 2}+C_{2} \delta_{4}^{2} y^{\prime 2}+1\right] \\
& {\left[C_{3} \sigma_{1}+C_{3} y^{2}+2 C_{3} y-2 C_{3} h_{0} y+1\right] }
\end{aligned}
$$

where

$$
\begin{aligned}
& Q_{3}=\mathrm{H}\left(f_{x}\right) \operatorname{sgn}\left(v_{f}\right) Q_{1}, \quad \sigma_{1}=h_{0}^{2}-2 h_{0}+1, \quad \sigma_{2}=v_{0}^{2}-2 v_{0}+1, \\
& \delta_{1}=v_{0}-1, \quad \delta_{2}=R_{0} C_{4}, \quad \delta_{3}=2 R_{0} C_{4}\left(v_{0}-1\right), \quad \delta_{4}=R_{0}\left(C_{4} v_{0}^{2}-2 C_{4} v_{0}+C_{4}+1\right) .
\end{aligned}
$$

Equation (4) cannot be used directly as it stands, and needs further expansion of the righthand side. However, this is problematic because of the large number of terms that are generated when this is done. To combat this a perturbation ordering scheme is proposed at this stage. The ordering procedure is based upon introducing an (assumed small) perturbation parameter $\varepsilon$ into the machining constants $C_{1}, C_{2}, C_{3}$, and $C_{4}$, and also into the two damping parameters for the tool, $\xi_{x}$ and $\xi_{y}$. It is relevant to note that this automatically assists in the definitions of certain grouped constants into single constant terms $P_{1}$ and $P_{2}$. The outcome of this is that terms of order greater than $O\left(\varepsilon^{1}\right)$ disappear from equation (4) and all remaining non-linear terms in that equation go to $O\left(\varepsilon^{1}\right)$. No higher order terms are generated by also applying this procedure to equation (3), so all terms from that equation are naturally retained. The physical basis for this ordering protocol is that the effect of the numerical values used for $C_{1}, C_{2}, C_{3}$, and $C_{4}$ (see reference [23]), when coupled with other terms, is to make them appear as relatively small terms to $O\left(\varepsilon^{2}\right)$, or higher order, in the differential equations. It is important to appreciate that the parameters $C_{1}, C_{2}, C_{3}$, and $C_{4}$ influence the functions $f_{x}$ and $f_{y}$ and that these parameters are coupled with other parameters such as cutting velocity and cutting depth. The data used for $C_{1}, C_{2}, C_{3}$, and $C_{4}$ has been taken from the previously published work of Grabec [5], and Wiercigroch [23], both of which were reliant on experimental data. The form of the resulting expansion of equation (4) is also far more algebraically manageable than it otherwise would have been. Damping can be assumed to be to $O\left(\varepsilon^{1}\right)$, in line with experimental findings. The additional grouped constants (denoted by the overbars) are defined in an attempt to tidy up the right-hand side terms in both differential equations, 
whilst also retaining explicit ordering for the perturbation analysis that comes next,

$$
\begin{gathered}
C_{1}=\varepsilon \overline{C_{1}}, \quad C_{2}=\varepsilon \overline{C_{2}}, \quad C_{3}=\varepsilon \overline{C_{3}}, \quad \xi_{x}=\varepsilon v_{x}, \quad \xi_{y}=\varepsilon v_{y}, \\
P_{1}=\varepsilon \overline{P_{1}}=\left[-2 C_{3} Q_{3} h_{0}\left(1-h_{0}\right)+C_{1} Q_{3} \sigma_{2}+C_{3} Q_{3} \sigma_{1}+C_{2} Q_{3} v_{0}^{2}-2 C_{2} Q_{3} v_{0}+C_{2} Q_{3}\right], \\
P_{2}=\varepsilon \overline{P_{2}}=\left[C_{1} Q_{3} h_{0} \sigma_{2}+C_{3} Q_{3} h_{0} \sigma_{1}+C_{2} Q_{3} h_{0} v_{0}^{2}-2 C_{2} Q_{3} h_{0} v_{0}+C_{2} Q_{3} h_{0}\right], \\
\overline{\gamma_{1}}=2 Q_{1} h_{0} \overline{C_{1}}\left(v_{0}-1\right), \quad \overline{\gamma_{2}}=Q_{1} \overline{C_{1}}\left(v_{0}-1\right)^{2}, \quad \overline{\gamma_{3}}=Q_{1} h_{0} \overline{C_{1}}, \\
\overline{\gamma_{4}}=Q_{1} \overline{C_{1}}, \quad \overline{\gamma_{5}}=2 Q_{1} \overline{C_{1}}\left(v_{0}-1\right), \quad \overline{\lambda_{1}}=2 \overline{C_{2}} Q_{3} h_{0} R_{0}\left(1-v_{0}\right), \\
\overline{\lambda_{2}}=\overline{C_{2}} Q_{3} h_{0} R_{0}^{2}, \quad \overline{\lambda_{3}}=\overline{C_{1}} Q_{3}, \quad \overline{\lambda_{4}}=2 \overline{C_{1}} Q_{3}\left(1-v_{0}\right), \\
\overline{\lambda_{5}}=\overline{C_{3}} Q_{3}, \quad \overline{\lambda_{6}}=2 \overline{C_{2}} Q_{3} R_{0}\left(1-v_{0}\right), \quad \overline{\lambda_{7}}=\overline{C_{2}} Q_{3} R_{0}^{2}, \quad \overline{\lambda_{8}}=\overline{C_{1}} Q_{3} h_{0}, \\
\overline{\lambda_{9}}=2 \overline{C_{1}} Q_{3} h_{0}\left(1-v_{0}\right), \quad \overline{\lambda_{10}}=\overline{C_{3}} Q_{3}\left(3 h_{0}-2\right), \quad \overline{\lambda_{11}}=Q_{3} h_{0} .
\end{gathered}
$$

Equations (5) and (6), given below, are the final forms of the system equations and imply a perturbation scheme of up to and including first order $\varepsilon$. Substitution of the above forms lead to the emergence of these two structurally simplified equations of motion for the system,

$$
\begin{gathered}
x^{\prime \prime}+x=-\varepsilon\left[2 v_{x}+\overline{\gamma_{1}}\right] x^{\prime}-\varepsilon \overline{\gamma_{2}} y+\varepsilon \overline{\gamma_{3}} x^{\prime 2}-\varepsilon \overline{\gamma_{4}} x^{\prime 2} y+\varepsilon \overline{\gamma_{5}} x^{\prime} y+\varepsilon^{0} Q_{2}-\varepsilon^{0} Q_{1} y \\
y^{\prime \prime}+\left(\alpha+Q_{3}\right) y=\varepsilon\left[-2 v_{y} \sqrt{\alpha}+\overline{\lambda_{1}}\right] y^{\prime}-\varepsilon \bar{P}_{1} y+\varepsilon \overline{\lambda_{2}} y^{\prime 2}-\varepsilon \overline{\lambda_{3}} x^{\prime 2} y-\varepsilon \overline{\lambda_{4}} x^{\prime} y-\varepsilon \overline{\lambda_{5}} y^{3}-\varepsilon \overline{\lambda_{6}} y y^{\prime} \\
-\varepsilon \overline{\lambda_{7}} y y^{\prime 2}+\varepsilon \overline{\lambda_{8}} x^{\prime 2}+\varepsilon \overline{\lambda_{9}} x^{\prime}+\varepsilon \overline{\lambda_{10}} y^{2}+\varepsilon \overline{P_{2}}+\varepsilon \overline{\lambda_{11}} .
\end{gathered}
$$

The consequence of neglecting terms from the right-hand side of equation (4) to get equation (6) has now to be discussed despite the implication of relative smallness of these terms by the chosen ordering protocol. The right-hand side function $f_{x}$ in equation (1) should be compared with the right-hand side of equation (5) (but with the term $2 v_{x}$ removed), and, correspondingly, function $f_{y}$ in equation (2) should be compared with the right-hand side of equation (6) (noting that the term $-Q_{3} y$ must be added and term $-2 v \sqrt{\alpha}$ must be removed). On that basis the functions describing the cutting process can be compared for the full and the simplified equations. This has been investigated for many parameter values with little difference evident for those cases. It was decided from thereon to restrict the comparison in the paper to the case based on the data of Grabec [4, 5] and Wiercigroch [23]. In Figures 2(a) and 2(b) the solid curves represent the analytical results obtained from the multiple scales analysis, whereas the dotted curves are obtained from numerical integration of the original equations (1) and (2). This shows that there are no visible differences between the two forms for $f_{x}$ and only the most minor differences evident between the two forms for $f_{y}$.

This is for typical cutting data and also for the two forces as functions of $v_{r}$ and $v_{f}$, respectively (Figures 2(a) and 2(b) and then as functions of $h$ (Figures 2(c) and 2(d). This provides sufficient motivation to proceed with equation (6) as an adequate, and much more practical, representation of equation (2).

\section{PERTURBATION ANALYSIS}

The method of multiple scales [37-39] is now so well established that little background explanation is needed other than to confirm that it uses the concept of an infinite series solution with successively higher order terms in the series offering non-linear corrections to the principal part, normally the lowest order linear solution. In this case the series is truncated after the first order term in $\varepsilon$, a decision forced by the absence of terms to higher order $\varepsilon$ in either of the differential equations (5) and (6). Therefore the solutions for $x$ and 


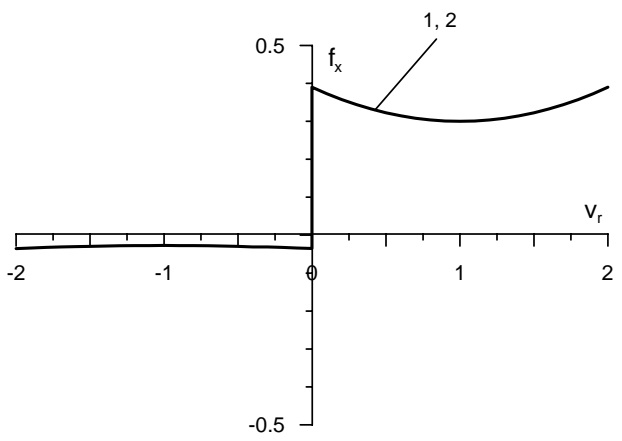

(a)

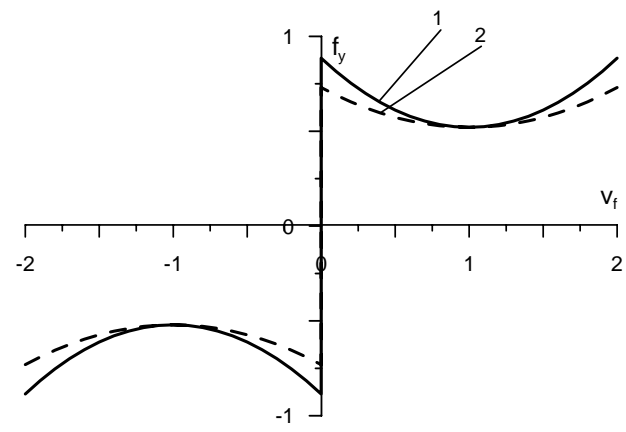

(b)

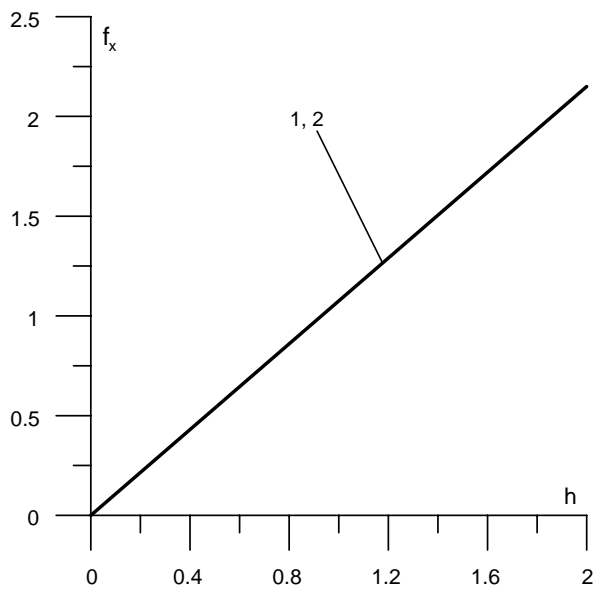

(c)

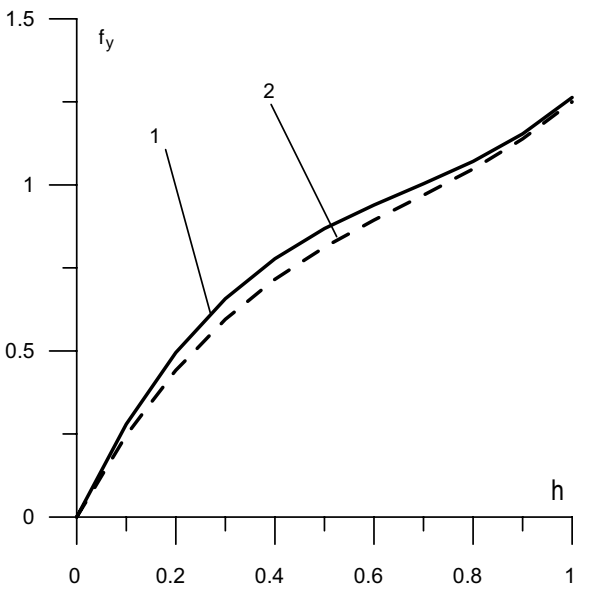

(d)

Figure 2. (a) Plot of force $f_{x}$ as a function of the relative velocity between the tool and the work-piece in the $x$ direction, $v_{r}$, for the full force term as stated in the right-hand side of equation (1)-curve 1 , and the approximation as calculated from the right-hand side of equation (5) - curve 2 (approximation and full term are indistinguishable). Additional data is, $\mu_{0}=0 \cdot 1, C_{1}=0 \cdot 3, C_{2}=0 \cdot 7, C_{3}=1 \cdot 5, C_{4}=1 \cdot 2, h_{0}=0 \cdot 5, R_{0}=2 \cdot 2, \alpha=4$. (b) Plot of force $f_{y}$ as a function of the relative velocity between the tool and the work-piece in the $y$ direction, $v_{f}$, for the full force term as stated in the right-hand side of equation (2)-curve 1, and the approximation as calculated from the right-hand side of equation (6)-curve 2. Additional data is, $\mu_{0}=0 \cdot 1, C_{1}=0 \cdot 3, C_{2}=0 \cdot 7$, $C_{3}=1.5, C_{4}=1 \cdot 2, h_{0}=0.5, R_{0}=2 \cdot 2, \alpha=4$. (c) Plot of force $f_{x}$ as a function of the actual cutting depth, $h$, for the full force term as stated in the right-hand side of equation (1)-line 1, and the approximation as calculated from the right-hand side of equation (5) - line 2 (approximation and full term are indistinguishable). Additional data is, $\mu_{0}=0.1, C_{1}=0.3, C_{2}=0.7, C_{3}=1.5, C_{4}=1.2, h_{0}=0.5, R_{0}=2 \cdot 2, \alpha=4$. (d) Plot of force $f_{y}$ as a function of the actual cutting depth, $h$, for the full force term as stated in the right-hand side of equation (2)-curve 1 , and the approximation as calculated from the right-hand side of equation (6)-curve 2. Additional data is, $\mu_{0}=0 \cdot 1, C_{1}=0 \cdot 3, C_{2}=0 \cdot 7, C_{3}=1 \cdot 5, C_{4}=1 \cdot 2, h_{0}=0 \cdot 5, R_{0}=2 \cdot 2, \alpha=4$.

$y$ are given by

$$
x=x_{0}+\varepsilon x_{1}+\ldots, \quad y=y_{0}+\varepsilon y_{1}+\ldots .
$$

Following the method the first and second time derivatives are also perturbed as follows:

$$
\frac{\mathrm{d}}{\mathrm{d} \tau}=\frac{\partial}{\partial T_{0}}+\varepsilon \frac{\partial}{\partial T_{1}}+\ldots=D_{0}+\varepsilon D_{1}+\ldots, \quad \frac{\mathrm{d}^{2}}{\mathrm{~d} \tau^{2}}=D_{0}^{2}+2 \varepsilon D_{0} D_{1}+\ldots
$$


The perturbed derivatives explicitly introduce new independent variables (timescales), $T_{0}$ and $T_{1}$, such that $x=x\left(T_{0}, T_{1}\right)$ and $y=y\left(T_{0}, T_{1}\right)$ and the subscripts 0 and 1 are used in the perturbation equations to show appropriate dependency on these two timescales. Separation of terms to $O\left(\varepsilon^{0}\right)$ and $O\left(\varepsilon^{1}\right)$ leads to two perturbation equations for $x$ and also for $y$, thus,

$$
\begin{aligned}
& 0\left(\varepsilon^{0}\right): \quad D_{0}^{2} x_{0}+x_{0}=Q_{2}-Q_{1} y_{0} \\
& 0\left(\varepsilon^{1}\right): \quad D_{0}^{2} x_{1}+x_{1}=-2 D_{0} D_{1} x_{0}-\left[2 v_{x}+\overline{\gamma_{1}}\right] D_{0} x_{0}-\overline{\gamma_{2}} y_{0} \\
&+\overline{\gamma_{3}}\left(D_{0} x_{0}\right)^{2}-\overline{\gamma_{4}}\left(D_{0} x_{0}\right)^{2} y_{0}+\overline{\gamma_{5}}\left(D_{0} x_{0}\right) y_{0}-Q_{1} y_{1} \\
& 0\left(\varepsilon^{0}\right): \quad D_{0}^{2} y_{0}+\left(\alpha+Q_{3}\right) y_{0}=\overline{\lambda_{11}} \\
& 0\left(\varepsilon^{1}\right): \quad D_{0}^{2} y_{1}+\left(\alpha+Q_{3}\right) y_{1}=-2 D_{0} D_{1} y_{0}+\left[-2 v_{y} \sqrt{\alpha}+\overline{\lambda_{1}}\right]\left(D_{0} y_{0}\right) \\
&-\bar{P}_{1} y_{0}+\overline{\lambda_{2}}\left(D_{0} y_{0}\right)^{2}-\overline{\lambda_{3}}\left(D_{0} x_{0}\right)^{2} y_{0}-\overline{\lambda_{4}}\left(D_{0} x_{0}\right) y_{0}-\overline{\lambda_{5}} y_{0}^{3} \\
&-\overline{\lambda_{6}}\left(D_{0} y_{0}\right) y_{0}-\overline{\lambda_{7}}\left(D_{0} y_{0}\right)^{2} y_{0} \\
&+\overline{\lambda_{8}}\left(D_{0} x_{0}\right)^{2}+\overline{\lambda_{9}}\left(D_{0} x_{0}\right)+\overline{\lambda_{10}} y_{0}^{2}+\overline{P_{2}}
\end{aligned}
$$

where

$$
\omega_{x}^{2}=1, \quad \omega_{y}^{2}=\left(\alpha+Q_{3}\right), \quad v_{1}=v_{x}, \quad v_{2}=v_{y} \sqrt{\alpha},
$$

It can be readily seen that the zeroth order perturbation equations (11) and (13) are inhomogeneous, but they are linear equations and can therefore be solved using conventional ordinary linear differential equation theory. In fact, it is simpler to start with equation (13) so that this solution for $y_{0}$ can then be used to construct the solution for $x_{0}$. The solutions for $x_{0}$ and $y_{0}$ are as follows, given here in complex exponential form,

$$
\begin{gathered}
x_{0}=A_{1} \mathrm{e}^{\mathrm{i} \omega_{x} T_{0}}+\overline{A_{1}} \mathrm{e}^{-\mathrm{i} \omega_{x} T_{0}}+K_{1}\left(A_{2} \mathrm{e}^{\mathrm{i} \omega_{y} T_{0}}+\overline{A_{2}} \mathrm{e}^{-\mathrm{i} \omega_{y} T_{0}}\right)+K_{2}+K_{1} K_{3}, \\
y_{0}=A_{2} \mathrm{e}^{\mathrm{i} \omega_{y} T_{0}}+\overline{A_{2}} \mathrm{e}^{-\mathrm{i} \omega_{y} T_{0}}+K_{3},
\end{gathered}
$$

where

$$
K_{1}=\frac{Q_{1}}{\left(\omega_{x}^{2}-\omega_{y}^{2}\right)}, \quad K_{2}=\frac{Q_{2}}{\omega_{x}^{2}}+\frac{K_{1} \lambda_{11}}{\omega_{x}^{2}}, \quad K_{3}=\frac{\lambda_{11}}{\omega_{y}^{2}} .
$$

Complex amplitudes $A_{1}$ and $A_{2}$ can be stated in terms of amplitudes $a_{1}$ and $a_{2}$ and phases $\phi_{1}$ and $\phi_{2}$ by means of equations (17) and (18)

$$
A_{1}=\frac{1}{2} a_{1} \mathrm{e}^{\mathrm{i} \phi_{1}}, \quad A_{2}=\frac{1}{2} a_{2} \mathrm{e}^{\mathrm{i} \phi_{2}},
$$

From this the zeroth order perturbation solutions can be recast in trigonometrical form, if required at this stage,

$$
\begin{gathered}
x_{0}=a_{1} \cos \left(\omega_{x} T_{0}+\phi_{1}\right)+K_{1} a_{2} \cos \left(\omega_{y} T_{0}+\phi_{2}\right)+K_{2}+K_{1} K_{3}, \\
y_{0}=a_{2} \cos \left(\omega_{y} T_{0}+\phi_{2}\right)+K_{3} .
\end{gathered}
$$

For the purposes of generating the first order perturbation corrections (i.e., $x_{1}$ and $x_{2}$ ) it is more convenient to continue using the zeroth order perturbation solutions in the form of equations (15) and (16). These are appropriately substituted into equations (12) and (14) and then resonance conditions are identified from the exponents of the resulting terms in 
the usual manner. The most interesting result is the non-resonant case because this is found between all the various resonant cases and must therefore occur for the widest range of machining conditions. Five internal resonance conditions can also be readily identified as, $\omega_{y} \approx 3 \omega_{x}, \omega_{y} \approx 2 \omega_{x}, \omega_{y} \approx \omega_{x}, \omega_{x} \approx 2 \omega_{y}$, and $\omega_{x} \approx 3 \omega_{y}$. There are no external resonances because this is a self-excitation problem that does not involve explicit external excitations. Because the non-resonant case is obviously of very particular practical interest it is examined exclusively in the remainder of this paper. In order to get to this point a more detailed consideration of the first order perturbation equations is necessary. Returning to equations (12) and (14), the two zeroth order solutions can be substituted in (from equations (15) and (16)) and differentiations with respect to $T_{0}$ carried out at the same time, to lead to new forms for the first order perturbation equations. The two first order perturbation equations contain very large numbers of terms and are therefore not shown here due to space restrictions. In fact specialized symbolic computer software [33, 34] has been employed throughout in order to do the complete multiple scales analysis. This is implemented within the Mathematica computer algebra system in the form of a new package called MultipleScales and has ensured the accuracy of the solution. All the principal results for the non-resonant case of interest are quoted from the formal output generated by this semi-automated approach.

Therefore, by continuing to apply the method of multiple scales all possible resonance conditions can be identified by inspection of the expanded right-hand sides of the first order perturbation equations. This leads to the immediate identification of secular terms (i.e., those terms that will invalidate the uniformity of the power series solutions to $x$ and $y$ ). As stated above, the fully expanded forms of the first order perturbation equations are not quoted explicitly, however the secular terms are shown for the interesting non-resonant condition, and are defined in the paper as solvability conditions, according to the accepted terminology of the method. From this it is then shown how the particular solutions to $x_{1}$ and $y_{1}$ can be constructed for the stable non-resonant case, on the understanding that this is the trivial solution from the point of view of the vibration analysis but the most desirable condition from the point of view of the machining process. The complete solutions for $x$ and $y$ under the stable non-resonant conditions are subsequently obtained by using the original series solutions given in equations (7) and (8).

\subsection{ANALYSIS OF THE NON-RESONANT CASE}

The solvability conditions for this case are obtained from the right-hand sides of the first order perturbation equations and are as follows:

$$
\frac{\mathrm{i} A_{1} \omega_{x}\left(\gamma_{1} \omega_{x}^{2}-K_{3} \gamma_{5} \omega_{x}^{2}+2 v_{1} \omega_{x}^{2}-\gamma_{1} \omega_{y}^{2}+K_{3} \gamma_{5} \omega_{y}^{2}-2 v_{1} \omega_{y}^{2}+K_{3} Q_{1} \lambda_{4}-Q_{1} \lambda_{9}\right)}{\left(\omega_{x}-\omega_{y}\right)\left(\omega_{x}-\omega_{y}\right)}+2 \mathrm{i} \omega_{x} A_{1}^{\prime}=0,
$$

$$
\begin{aligned}
& \bar{A}_{2}\left(K_{1}^{2} \lambda_{3} \omega_{y}^{2}+\lambda_{7} \omega_{y}^{2}+3 \lambda_{5}\right) A_{2}^{2}+\left(3 \lambda_{5} K_{3}^{2}-2 \lambda_{10} K_{3} \mathrm{i} K_{2} \lambda_{4} \omega_{y} K_{3}\right. \\
& \left.+\mathrm{i} \lambda_{6} \omega_{y} K_{3}+2 \bar{A}_{1} A_{1} \lambda_{3} \omega_{x}^{2}+P_{1}-\mathrm{i} \lambda_{1} \omega_{y}-\mathrm{i} K_{1} \lambda_{9} \omega_{y}+2 \mathrm{i} v_{2} \omega_{y}\right) A_{2}+2 \mathrm{i} \omega_{y} A_{2}^{\prime}=0,
\end{aligned}
$$

noting that they are still in terms of complex amplitudes $A_{1}$ and $A_{2}$. Substitution of equations (17) and (18) for these complex amplitudes in equations (21) and (22), and then separation of terms into real and imaginary parts, yields four modulation equations, as stated in Appendix A, (equations (A.1)-(A.4) inclusive). These modulation equations are readily solved and lead to the following solutions for $a_{1}, a_{2}, \phi_{1}$, and $\phi_{2}$, noting that $c[1]$, 
$c[2]$, and $c[3]$ are constants of integration,

$$
\begin{aligned}
& a_{1}=c[3] \exp \left(-\frac{K_{3} Q_{1} \lambda_{4}}{2\left(\omega_{x}-\omega_{y}\right)\left(\omega_{x}-\omega_{y}\right)}+\frac{Q_{1} \lambda_{9}}{2\left(\omega_{x}-\omega_{y}\right)\left(\omega_{x}+\omega_{y}\right)}\right. \\
& -\frac{\gamma_{1} \omega_{x}^{2}}{2\left(\omega_{x}-\omega_{y}\right)\left(\omega_{x}+\omega_{y}\right)}+\frac{K_{3} \gamma_{5} \omega_{x}^{2}}{2\left(\omega_{x}-\omega_{y}\right)\left(\omega_{x}+\omega_{y}\right)}-\frac{v_{1} \omega_{x}^{2}}{\left(\omega_{x}-\omega_{y}\right)\left(\omega_{x}+\omega_{y}\right)} \\
& \left.+\frac{\gamma_{1} \omega_{y}^{2}}{2\left(\omega_{x}-\omega_{y}\right)\left(\omega_{x}+\omega_{y}\right)}-\frac{K_{3} \gamma_{5} \omega_{y}^{2}}{2\left(\omega_{x}-\omega_{y}\right)\left(\omega_{x}+\omega_{y}\right)}+\frac{v_{1} \omega_{y}^{2}}{\left(\omega_{x}-\omega_{y}\right)\left(\omega_{x}+\omega_{y}\right)}\right) t \\
& a_{2}=c[2] \exp \left(\frac{\lambda_{1}}{2}-\frac{1}{2} K_{1} K_{3} \lambda_{4}-\frac{K_{3} \lambda_{6}}{2}+\frac{K_{1} \lambda_{9}}{2}-v_{2}\right) t \\
& \phi_{1}=\phi_{1}(0) \\
& \phi_{2}=c[1]+\frac{\left(P_{1}+3 K_{3}^{2} \lambda_{5}-2 K_{3} \lambda_{10}\right) t}{2 \omega_{y}}+\frac{\mathrm{e}^{\left(\lambda_{1}-K_{1} K_{3} \lambda_{4}-K_{3} \lambda_{6}+K_{1} \lambda_{9}-2 v_{2}\right) t} c[2]^{2}\left(3 \lambda_{5}+K_{1}^{2} \lambda_{3} \omega_{y}^{2}+\lambda_{7} \omega_{y}^{2}\right)}{8\left(\lambda_{1}-K_{1} K_{3} \lambda_{4}-K_{3} \lambda_{6}+K_{1} \lambda_{9}-2 v_{2}\right) \omega_{y}} \\
& +\exp \left(\frac{\left(K_{3} Q_{1} \lambda_{4}-Q_{1} \lambda_{9}+\gamma_{1} \omega_{x}^{2}-K_{3} \gamma_{5} \omega_{x}^{2}+2 v_{1} \omega_{x}^{2}-\gamma_{1} \omega_{y}^{2}+K_{3} \gamma_{5} \omega_{y}^{2}-2 v_{1} \omega_{y}^{2}\right) t}{\left(-\omega_{x}+\omega_{y}\right)\left(\omega_{x}+\omega_{y}\right)}\right) \\
& c[3]^{2} \lambda_{3} \omega_{x}^{2}\left(-\omega_{x}+\omega_{y}\right)\left(\omega_{x}+\omega_{y}\right) / \\
& \left(4 \omega_{y}\left(K_{3} Q_{1} \lambda_{4}-Q_{1} \lambda_{9}+\gamma_{1} \omega_{x}^{2}-K_{3} \gamma_{5} \omega_{x}^{2}+2 v_{1} \omega_{x}^{2}-\gamma_{1} \omega_{y}^{2}+K_{3} \gamma_{5} \omega_{y}^{2}-2 v_{1} \omega_{y}^{2}\right)\right) .
\end{aligned}
$$

The constants of integration can be determined from the initial displacement and velocity conditions for $x$ and $y$, however because of the particular structure of equations (23)-(26) it is much easier to solve the inverse problem in which one proceeds from assumed constants to calculate corresponding initial conditions. An iterative calculation can then be used to determine the constants for a desired set of initial conditions. It is important to appreciate that as time approaches infinity the amplitudes $a_{1}$ and $a_{2}$ will tend to zero if the overall value of the constant part of the exponents of equations (23) and (24) is negative. In other words, if

$$
-\frac{K_{3} Q_{1} \lambda_{4}-Q_{1} \lambda_{9}+\gamma_{1} \omega_{x}^{2}-K_{3} \gamma_{5} \omega_{x}^{2}+2 v_{1} \omega_{x}^{2}-\gamma_{1} \omega_{y}^{2}+K_{3} \gamma_{5} \omega_{y}^{2}-2 v_{1} \omega_{y}^{2}}{2\left(\omega_{x}-\omega_{y}\right)\left(\omega_{x}+\omega_{y}\right)}<0 .
$$

and

$$
\frac{1}{2}\left(\lambda_{1}-K_{1} K_{3} \lambda_{4}-K_{3} \lambda_{6}+K_{1} \lambda_{9}-2 v_{2}\right)<0 .
$$

Inequalities (27) and (28) do not contain the constants of integration and are therefore independent of the initial conditions, as, therefore, are the conditions for stability. If inequalities (27) and (28) are not satisfied then the amplitudes will grow exponentially with time, and are definitionally unstable. Assuming that inequalities (27) and (28) are satisfied, and this implies conditions for stability, then the particular solutions to the first order perturbations can be obtained, notwithstanding the somewhat tedious algebra required. This is also handled by the symbolic solution software [33, 34], to give

$$
\begin{gathered}
x_{1}=\frac{-P_{2} Q_{1}+K_{3}^{3} Q_{1} \lambda_{5}-K_{3}^{2} Q_{1} \lambda_{10}+K_{3}\left(P_{1} Q_{1}-\gamma_{2} \omega_{y}^{2}\right)}{\omega_{x}^{2} \omega_{y}^{2}}, \\
y_{1}=\frac{-K_{3} P_{1}+P_{2}-K_{3}^{3} \lambda_{5}+K_{3}^{2} \lambda_{10}}{\omega_{y}^{2}} .
\end{gathered}
$$


These solutions for $x_{1}$ and $y_{1}$, together with those for $x_{0}$ and $y_{0}$, can be substituted into equations (7) and (8) to give the complete solutions for $x$ and $y$ for the non-resonant case, on the understanding that inequalities (27) and (28) hold true. The solutions for $x_{0}$ and $y_{0}$ will obviously equal $\left(K_{2}+K_{1} K_{3}\right)$, and $K_{3}$, respectively, given that $a_{1}(\infty)=a_{2}(\infty)=0$ in this case. From this it can be seen that the stable, non-resonant, solutions for $x$ and $y$ are constant valued dc offsets. These are the trivial solutions for the vibration analysis, as discussed above, but highly desirable as far as the machining process is concerned. In order to examine the effect of these conditions in practice inequalities (27) and (28) can be restated in terms of the original physical parameters, thus,

$$
\begin{gathered}
\frac{C_{1} h_{0} q_{0}^{2}\left(-1+v_{0}\right) \alpha-\sqrt{\alpha}\left(-1+q_{0}+\alpha\right)\left(C_{2} h_{0} q_{0} R_{0}\left(-1+v_{0}\right) \sqrt{\alpha}+\left(q_{0}+\alpha\right) \xi_{y}\right)}{\varepsilon\left(-1+q_{0}+\alpha\right)\left(q_{0}+\alpha\right)}<0, \\
\frac{C_{1} h_{0} q_{0}^{2}\left(-1+v_{0}+\alpha-\alpha v_{0}\right) \alpha+\left(q_{0}-q_{0}^{2}+\alpha-2 q_{0} \alpha-\alpha^{2}\right) \xi_{x}}{\varepsilon\left(-1+\sqrt{q_{0}+\alpha}\right)\left(1+\sqrt{q_{0}+\alpha}\right)\left(q_{0}+\alpha\right)}<0,
\end{gathered}
$$

noting that these are for full contact conditions defined by $\operatorname{sgn}()=1$ and $H()=1$, in the limit of relatively small oscillations. If inequalities (31) and (32) are satisfied then they can be rearranged to show that the work-piece velocity, $v_{0}$, must satisfy both the following conditions for stability:

$$
\begin{gathered}
v_{01}>-\frac{C_{1} h_{0} q_{0}^{2} \alpha-\sqrt{\alpha}\left(-1+q_{0}+\alpha\right)\left(C_{2} h_{0} q_{0} R_{0} \sqrt{\alpha}+\left(q_{0}+\alpha\right) \xi_{y}\right)}{C_{1} h_{0} q_{0}^{2} \alpha-C_{2} h_{0} q_{0} R_{0} \alpha\left(-1+q_{0}+\alpha\right)}, \\
v_{02}>-\frac{C_{1} h_{0} q_{0}^{2}(-1+\alpha) \alpha-\left(q_{0}+\alpha\right)\left(1-q_{0}+\alpha\right) \xi_{x}}{C_{1} h_{0} q_{0}(1-\alpha) \alpha} .
\end{gathered}
$$

Figures 3(a) and 3(b) show plots of $v_{0}$ as functions of cutting force magnitude, $q_{0}$, and initial depth of cut, $h_{0}$, with the upper solid line delineating the theoretically stable region (white) from the theoretically unstable region (grey). The dotted line is the commensurate numerical solution for the upper solid line. Numerical simulations were carried out by means of the fourth order Runge Kutta method, and vibrations of the system were analyzed for small initial conditions. The dotted line in the figures divides the parameter plane into two regions with a stable regime predicted for the space above the line and unstable behaviour below. It can be seen in Figure 3(a) that there is close qualitative similarity between them but a fairly constant quantitative discrepancy of approximately $20 \%$. This is because the numerical solutions for $v_{0}$ (versus $q_{0}$ and $h_{0}$ ) have been obtained from the original full equations (1) and (2), whereas the conditions in inequalities (33) and (34) emanate from the multiple scales analysis. As is already apparent the multiple scales analysis contains inherent approximations due to series truncation and the assumptions that accompany the use of solvability conditions in general, in addition to the special modelling simplifications that took place at the time of term ordering so as to ensure a reasonably tractable algebraic problem from thereon. This discrepancy is not consistent, or as significant, in Figure 3(b).

It is also instructive to examine the zeroth order perturbation solutions, $x_{0}$ and $y_{0}$ in the time domain, and two cases for each are presented in Figures 4 and 5. In Figure 4 solutions are given for $x_{0}$ and $y_{0}$ for a selected stable point as defined in Figure 3(b), where $q_{0}=1$, $h_{0}=0.5$, and $v_{0}=0.8$ (i.e. a point within the white region in Figure $3(\mathrm{~b})$ ). It can be seen that both responses decay with time to what will become levelled-out dc offsets a little later on. The first order perturbation corrections of equations (29) and (30) would add small corrections to these two responses. The converse situation is shown in Figure 5 where the operating reference point in Figure 3(b) is shifted into the unstable grey region, such that 

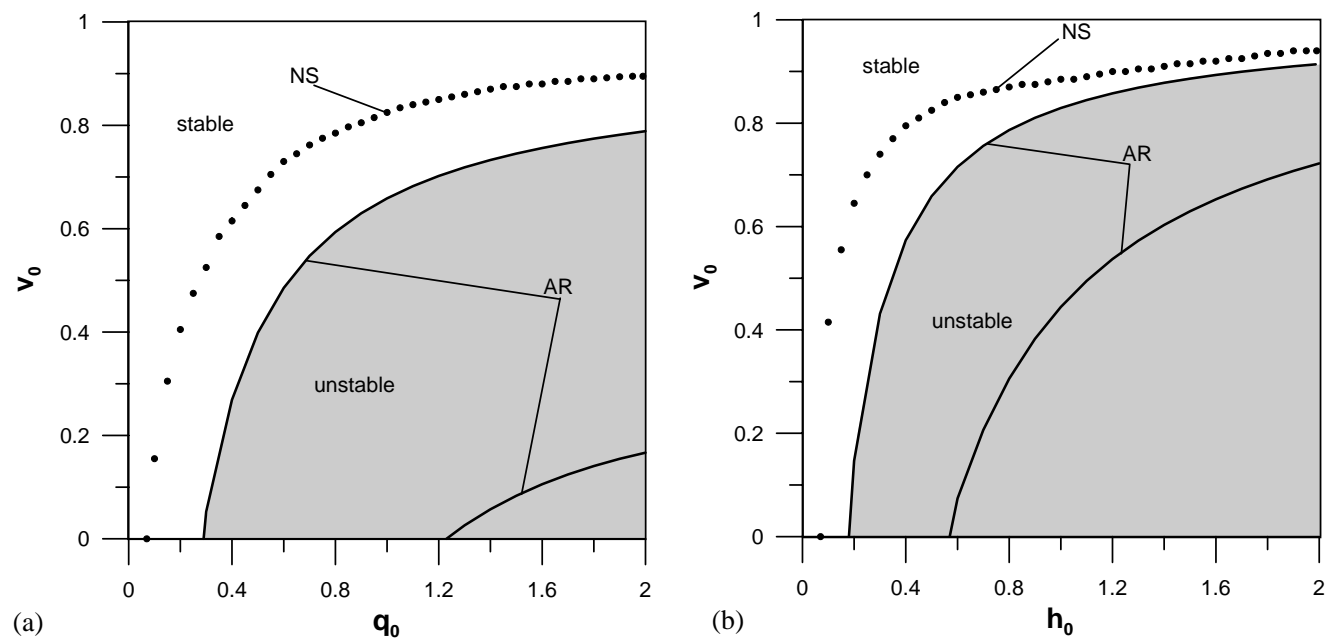

Figure 3. (a) Plot of feed velocity $v_{o}$ as a function of the magnitude of the cutting force $q_{0}$, for the multiple scales analysis (analytical result AR-solid curve) and for numerical analysis based on the original equations of motion due to Wiercigroch [23] (numerical solution NS-dotted curve). Stable area is white, unstable area is grey. Inner analytical solution is not necessary for the stability criterion. Additional data is, $\mu_{0}=0 \cdot 1, C_{1}=0 \cdot 3, C_{2}=$ $0 \cdot 7, C_{3}=1 \cdot 5, C_{4}=1 \cdot 2, h_{0}=0 \cdot 5, R_{0}=2.2, \alpha=4, \xi_{x}=0 \cdot 1, \xi_{y}=0 \cdot 1$. (b) Plot of feed velocity $v_{o}$ as a function of the initial depth of cut $h_{0}$, for the multiple scales analysis (analytical result AR-solid curve) and for numerical analysis based on the original equations of motion due to Wiercigroch [23] (numerical solution NS-dotted curve). Stable area is white, unstable area is grey. Inner analytical solution is not necessary for the stability criterion. Additional data is, $\mu_{0}=0 \cdot 1, C_{1}=0 \cdot 3, C_{2}=0 \cdot 7, C_{3}=1 \cdot 5, C_{4}=1 \cdot 2, R_{0}=2 \cdot 2, \alpha=4, \xi_{x}=0 \cdot 1, \xi_{y}=0 \cdot 1$.

$q_{0}=1, h_{0}=0 \cdot 5$, and $v_{0}=0 \cdot 4$. The time-domain plots for $x_{0}$ and $y_{0}$ are given in Figure 5 and show typical exponential growth with time, indicating instability. It is worth noting that the time response for $x_{0}$ is also subjected to a low frequency modulation, this being an additional, unexplored, interaction between the work-piece feed speed $v_{0}$ and the local selfexcitation due to the orthogonal interactions in $x$ and $y$.

\section{SUMMARY AND CONCLUSIONS}

This work has shown that it is possible to generate approximate analytical solutions to the non-resonant, self-excited, orthogonal metal cutting problem, as represented in a twodegree-of-freedom system in which dry friction chatter occurs. The multiple scales method as applied, has enabled an approximate analysis to be undertaken of the dynamic interactions occurring in the metal cutting process. This facilitates the provision of guidance on the selection of technological parameters for the avoidance of primary chatter. This paper looks, for the first time, at the predicted stability regions on the basis of a practically useful technological parameter space, involving the depth of cut and the cutting speed. The non-resonant case is of particular interest because it is necessarily a much more generally widespread, and therefore potentially dangerous, phenomenon than the specific resonances that are also predicted by the analysis. The solutions for the nonresonant case have been generated from structurally simplified equations of motion based on the seminal equations first postulated by Wiercigroch [23], although much of the original discontinuous behaviour of Wiercigroch's model is still retained within the simplified equations. The simplifications extend to a reduction in the complexity of the 


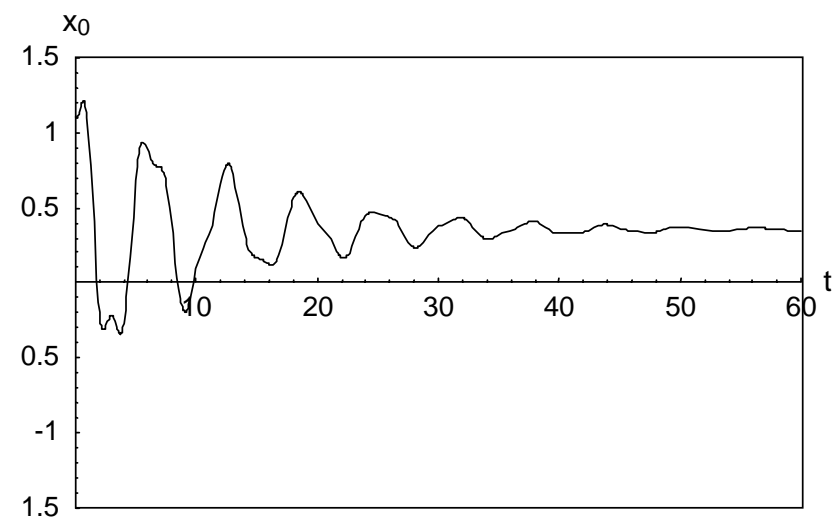

(a)

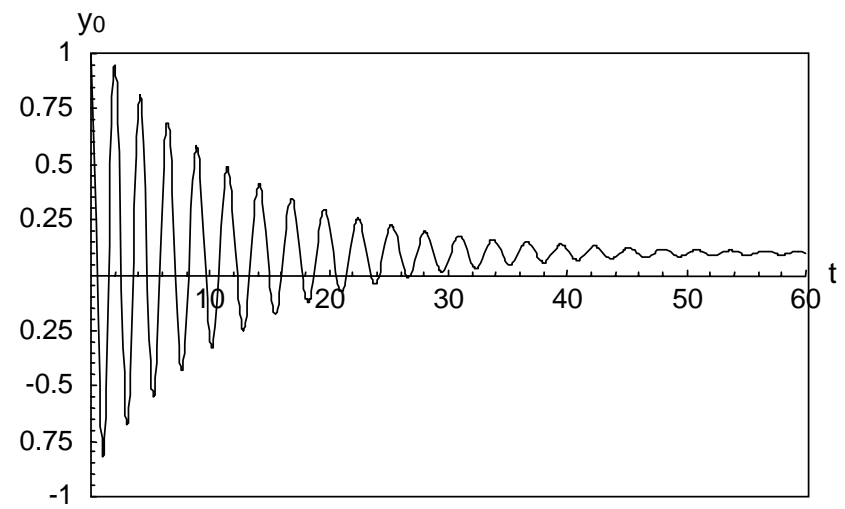

(b)

Figure 4. Time domain plots for $x_{0}$ (a) and $y_{0}$ (b) for stable cutting, for which $q_{0}=1, h_{0}=0 \cdot 5$, and $v_{0}=0 \cdot 8$. Additional data is, $\mu_{0}=0 \cdot 1, C_{1}=0.3, C_{2}=0 \cdot 7, C_{3}=1 \cdot 5, C_{4}=1 \cdot 2, R_{0}=2 \cdot 2, \alpha=4, \xi_{x}=0 \cdot 1, \xi_{y}=0 \cdot 1$. The integration constants used to calculate the time domain responses are $C[1]=C[2]=C[3]=1$, with $\phi(0)=0$, which equate to initial conditions $x(0)=1.43, \dot{x}(0)=-0.91, y(0)=-0.0065, \dot{y}(0)=3 \cdot 48$.

terms expressing the orthogonal cutting forces, $f_{x}$ and $f_{y}$. Although there are no discernible differences between the full and simplified forces in the $x$ direction, and little difference between their counterparts in the $y$ direction, there is around $20 \%$ difference between the feed-rate velocities predicted by the multiple scales analysis and those calculated numerically from the original equations of motion due to Wiercigroch [23]. This difference is largely attributable to an accumulation of errors starting from the structural simplifications to the equations of motion and then through using the approximate perturbation method of multiple scales. Regarding the specific resonance conditions that are also predicted by the multiple scales analysis, these involve relationships in the form of positive integers between the natural frequencies $\omega_{x}$ and $\omega_{y}$ which lead to additional forms of localised resonant behaviour.

The multiple scales perturbation analysis was carried out by using a specialized symbolically based computational solver, Khanin et al. [33] and Khanin and Cartmell [34], and has led to an analytical criterion, in the form of formulas (31) and (32), for the definition of stable, or unstable, dry friction chatter vibrations in orthogonal metal cutting. Compared with numerical methods, including neural network procedures, this is a 


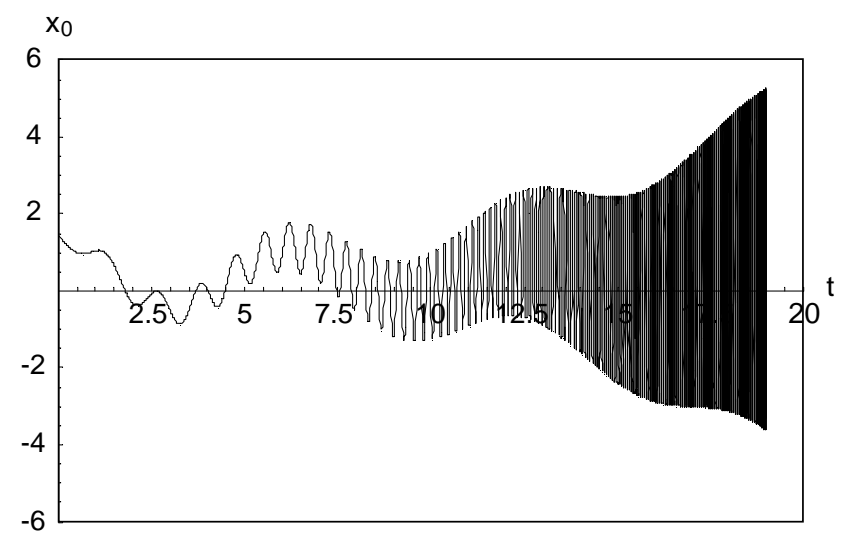

(a)

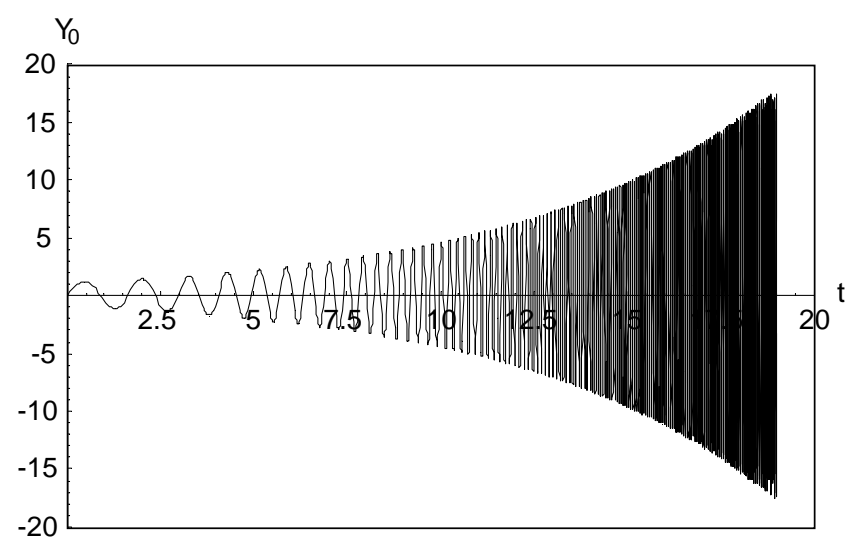

(b)

Figure 5. Time domain plots for $x_{0}$ (a) and $y_{0}$ (b) for unstable cutting, for which $q_{0}=1, h_{0}=0 \cdot 5$, and $v_{0}=0.4$. Additional data is, $\mu_{0}=0 \cdot 1, C_{1}=0 \cdot 3, C_{2}=0 \cdot 7, C_{3}=1 \cdot 5, C_{4}=1 \cdot 2, R_{0}=2 \cdot 2, \alpha=4, \xi_{x}=0 \cdot 1, \xi_{y}=0 \cdot 1$. The integration constants used to calculate the time domain responses are $C[1]=C[2]=C[3]=1$, with $\phi(0)=0$, which equate to initial conditions $x(0)=1.43, \dot{x}(0)=-0.91, \quad y(0)=-0.0065, \quad \dot{y}(0)=3.48$.

fast and elegant approach to such a criterion, although it is clear that a good dynamical model and physically realistic data are important pre-requisites.

It is also shown (in Figure 3(b)) that dry friction chatter stabilises for deep cutting at high feed velocities but destabilizes at low feed velocities.

There is also an, as yet, unexplored low-frequency modulation of the theoretically predicted time domain response in the $x$ direction for the unstable case, but it is thought that this is due to dynamic coupling between the work-piece feed and the local dynamics of the cutting tool, as modelled here.

Further work is planned in the form of experiments to be carried out at the Technical University of Lublin to determine stability/instability transitions against which the analytical predictions stated within this paper can be checked for a range of cutting parameter data. This will determine to what extent the analysis could be revised for higher levels of precision. The low frequency modulation that is predicted for the response of the tool in the $x$ direction in the unstable case is also to be investigated and reported in the literature in due course. The resonant phenomena predicted by the analysis will also be 
analyzed and reported as appropriate. A new programme of research on the use of the results in this paper for the real-time control of the cutting process is under consideration, and this relates to the recent work of Pratt and Nayfeh [40] on chatter control and stability analysis of a cantilever boring bar under regenerative cutting conditions. It is also intended that the work of this paper could be used to provide a map of stable cutting parameters for workshop use in the manual setting-up of machine-tools.

The results from this research should be adaptable to other machining processes such as turning or milling, and new work is planned to explore these other applications.

\section{ACKNOWLEDGMENTS}

The authors would like to acknowledge the financial support provided by the Polish State Committee for Scientific Research, grant number 126/E-361/SPUB/COST/T-7/DZ 42/99, the Polish Academy of Sciences, the British Council, and the Royal Society, for funding the collaborations which led to the completion of this work. They would also like to note the support provided by the UK's Engineering and Physical Sciences Research Council on grant GR/L30749 for the independent research which led to the development of the semi-automated symbolic solver package used for the multiple scales analysis in this research programme. The Royal Society Dorothy Hodgkin Research Fellowship awarded to R. Khanin at the University of Glasgow and then at the University of Cambridge, is also gratefully acknowledged. The authors would also like to thank Eng. B. Pawłowska for her help in compiling the manuscript.

\section{REFERENCES}

1. S. A. Tobias 1965 Machine Tool Vibration. New York: Wiley.

2. H. OpITZ and F. BERNARDI 1970 Annals of the CIRP 18, 335-343. Investigation and calculation of the chatter behaviour of lathes and milling machines.

3. J. Thusty 1986 American Society of Mechanical Engineers, Journal of Engineering for Industry 108, 59-67. Dynamics of high speed milling.

4. I. GRABEC 1986 Physics Letters A 117, 384-386. Chaos generated by the cutting process.

5. I. GRA BEC 1988 International Journal of Machine Tool Manufacture 28, 19-31. Chaotic dynamics of the cutting process.

6. E. Marui, S. Kato and M. Hashimoto 1988 American Society of Mechanical Engineers, Journal of Engineering for Industry 110, 236-241. The mechanism of chatter vibration in a spindleworkpiece system. Part 1 - properties of self-excited chatter vibration in a spindle-workpiece system 110, 242-247. The mechanism of chatter vibration in a spindle-workpiece system. Part 2-characteristics of dynamic cutting force and vibration energy 110, 248-253. The mechanism of chatter vibration in a spindle-workpiece system. Part 3-analytical considerations.

7. J. S. LiN and C. I. WENG 1990 International Journal of Machine Tool Manufacture 19, 53-64. A non-linear dynamic model of cutting.

8. J. S. Lin and C. I. Weng 1991 International Journal of Mechanical Sciences 33, 645-657. Nonlinear dynamics of the cutting process.

9. J. Gradisek, E. Govekar and I. Grabec 1996 International Journal of Machine Tool Manufacture 36, 1161-1172. A chaotic cutting process and determining optimal cutting parameter using neural networks.

10. J. Gradisek, E. Govekar and I. Grabec 1998 Mechanical Systems and Signal Processing 12, 839-854. Time series analysis in metal cutting: chatter versus chatter-free cutting.

11. J. Gradisek, E. GovekAR and I. Grabec 2001 Journal of Sound and Vibration 242, 829-838. Chatter onset in non-regenerative cutting: a numerical study.

12. G. Stepan and T. Kalmar-Nagy 1997 Proceedings of DETC '97, 16th ASME Biennial Conference on Mechanical Vibrations and Vibrations and Noise, September 14-17, Sacramento, CA, U.S.A., 1-11. Non-linear regenerative machine tool vibrations. 
13. G. LitAK, J. WARMiński and J. Lipski 1997 Proceedings of the 4th Conference on Dynamical Systems-Theory and Applications, Łódż, Poland, 8-9 December, (J. AwreJCEwicz, J. Gr ABSKI and J. Mrozowski, editors), 193-198. Self-excited vibrations in the cutting process.

14. W. Przystupa and G. LITAK 1998 Proceedings of the XXXVII Symposium on Modelling in Mechanics, Wista, Poland, 305-310. Cutting process in the presence of noise.

15. M. A. Johnson and F. C. Moon 2001 International Journal of Bifurcation and Chaos 11, 449-467. Non-linear techniques to characterise pre-chatter and chatter vibrations in the machining of metals.

16. G. Stepan 2001 Philosophical Transactions of the Royal Society of London A 359, 739-757. Modelling nonlinear regenerative effects in metal cutting.

17. M. Wiercigroch and E. BUdAK 2001 Philosophical Transactions of the Royal Society of London A 359, 663-693. Sources of non linearities, chatter generation, and suppression in metal cutting.

18. F. C. Moon, and T. Kalmar-Nagy 2001 Philosophical Transactions of the Royal Society of London A 359, 695-711. Non-linear models for complex dynamics in cutting materials.

19. E. Rabinowitz 1958 Proceedings of the Physical Society 71, 668-673. The intrinsic variables affecting the stick-slip process.

20. R. Bell and M. BuRdeKin 1970 Proceedings of the Institution of Mechanical Engineers 184, 543-557. A study of stick-slip motion of machine tool drives.

21. M. Wiercigroch 1989 Ph.D. Thesis, Silesian Technical University, Gliwice, Poland. Modelling of dynamic interaction between machine tool and cutting process (in Polish).

22. M. Wiercigroch and J. Kosmol 1993 GEP, XLV, 39-41. Active control of nonlinear vibrations of the MTCP system.

23. M. Wiercigroch 1997 Transactions of the American Society of Mechanical Engineers, Journal of Vibration and Acoustics 119, 468-475. Chaotic vibrations of a simple model of the machine tool—cutting process system.

24. M. Wiercigroch and A. H.-D. Cheng 1997 Chaos, Solitons, and Fractals 8, 715-726. Chaotic and stochastic dynamics of orthogonal metal cutting.

25. J. Warmiñski, G. Litak, J. Lipski, M. Wiercigroch and M. P. Cartmell 2000 in IUTAM/ IFToMM Symposium on Synthesis of Nonlinear Dynamical Systems (E. Lavendis and M. Zakrzhevsky, editors.), Dordrecht, Netherlands: Kluwer Academic Publishers. 275-284. Chaotic vibrations in the regenerative cutting process.

26. T. Kalmar-Nagy and J. R. Pratt 1999 Proceedings of DETC' 99, 17th Biennial Conference on Mechanical Vibration and Noise, Las Vegas, U.S.A., DETC99/VIB-8060. Experimental and analytical investigation of the subcritical instability in metal cutting.

27. M. Wiercigroch and A. Krivtsov 2001 Philosophical Transactions of the Royal Society of London A 359, 713-738. Frictional chatter in orthogonal metal cutting.

28. E. P. Nosyreva and A. Molinari 1998 International Journal of Mechanical Sciences 40, 735-748. Analysis of non-linear vibrations in metal cutting.

29. M. E. Merchant 1944 Transactions of the American Society of Mechanical Engineers, Journal of Applied Mechanics A 11, 168-175. Basic mechanics in the metal cutting process.

30. C.-C. Hwang, R.-F. Fung and J. S. Lin 1997 Journal of Sound and Vibration 203, 363-372. Strong non-linear dynamics of cutting process.

31. N. H. Hanna and S. A. Tobias 1974 Transactions of the American Society of Mechanical Engineers, Journal of Engineering for Industry 96, 247-255. A theory of nonlinear regenerative chatter.

32. A. H. Nayfeh and C.-M. Chin and J. Pratt 1997 Journal of Manufacturing Science and Engineering 195, 485-493. Perturbation methods in nonlinear dynamics-applications to machining dynamics.

33. R. Khanin, M. P. Cartmell and A. D. Gilbert 2000 Computers and Structures 76, 565-575. A computerised implementation of the multiple scales perturbation method using Mathematica.

34. R. Khanin and M. P. Cartmell 2001 Journal of Symbolic Computing 31, 461-473. Parallelisation of perturbation analysis: application to large-scale engineering problems.

35. F. Jiulian, Y. ZheJun and Y. YingXue 1989 International Journal of Machine Tool Manufacture 29, 601-609. A unified system model of cutting chatter and its transformation function.

36. W. F. Hastings, P. Mathew and P. L. B. Oxley 1980 Proceedings of the Royal Society of London A 371, 343-354. A machining theory for predicting chip geometry, cutting forces, etc. from material properties and cutting conditions. 
37. A. H. NaYfeh 1974 Perturbation Methods. New York: Wiley.

38. A. H. NAYFEH and D. T. Mook 1979 Nonlinear Oscillations. New York: Wiley.

39. M. P. Cartmell 1990 An Introduction to Linear, Parametric and Nonlinear Vibrations. London: Chapman \& Hall.

40. J. R. Pratt and A. H. NaYfeh 2001 Philosophical Transactions of the Royal Society of London A 359, 759-792. Chatter control and stability analysis of a cantilever boring bar under regenerative cutting conditions.

\section{APPENDIX A}

The modulation equation for the non-resonant case are as follows:

$$
-a_{1} \omega_{x} \phi_{1}^{\prime}=0
$$

$$
\begin{gathered}
a_{2}^{3}\left(\frac{3 \lambda_{5}}{8}+\frac{K_{1}^{2} \lambda_{3} \omega_{y}^{2}}{8}+\frac{\lambda_{7} \omega_{y}^{2}}{8}\right)+\frac{1}{4} a_{2}\left(2 P_{1}+6 K_{3}^{2} \lambda_{5}-4 K_{3} \lambda_{10}+a_{1}^{2} \lambda_{3} \omega_{x}^{2}-4 \omega_{y} \phi_{2}^{\prime}\right)=0 \\
a_{1} \omega_{x}\left(K_{3} Q_{1} \lambda_{4}-Q_{1} \lambda_{9}+\gamma_{1} \omega_{x}^{2}-K_{3} \gamma_{5} \omega_{x}^{2}+2 v_{1} \omega_{x}^{2}-\gamma_{1} \omega_{y}^{2}\right. \\
\left.+K_{3} \gamma_{5} \omega_{y}^{2}-2 v_{1} \omega_{y}^{2}\right) /\left(2\left(\omega_{x}-\omega_{y}\right)\left(\omega_{x}+\omega_{y}\right)\right)+\omega_{x} a_{1}^{\prime}=0 \\
-\frac{1}{2} a_{2}\left(\lambda_{1}-K_{1} K_{3} \lambda_{4}-K_{3} \lambda_{6}+K_{1} \lambda_{9}-2 v_{2}\right) \omega_{y}+\omega_{y} a_{2}^{\prime}=0
\end{gathered}
$$

